\title{
A pesquisa sobre redes sociais e comportamento do consumidor no Brasil
}

\section{Ana Cristina Ferreira}

Professora substituta da Universidade Federal de Viçosa-Campus de Rio Paranaíba. Mestra pelo Programa de Pós Graduação em Administração da Universidade Federal de Uberlândia (UFU). Bacharela em Administração pela Universidade Federal de Viçosa Campus de Rio Paranaíba (UFVCRP), Minas Gerais, Brasil ana-cristina18@hotmail.com

\section{André Francisco Alcântara Fagundes}

Professor do Programa de Pós-Graduação em Administração (PPGA) da Faculdade de Gestão e Negócios (FAGEN) da Universidade Federal de Uberlândia (UFU). Doutor em Administração pela Universidade Federal de Minas Gerais (UFMG). Mestre em Administração pela UFU, Minas Gerais, Brasil

andre.fagundes@ufu.br

Mariany Carvalho Silva Camargo

Pós-graduada em Finanças e Estratégias Empresariais (UFU). Graduada em Administração pela Universidade Federal de Uberlândia, Minas Gerais, Brasil mariany.silva@itau-unibanco.com.br

Thais Carvalho Oliveir

Mestrado em Administração (UFU), especialização em Gestão Pública Municipal (FAGEN/UFU) e em Supervisão Escolar (Faculdade Católica de Uberlândia), graduação em Geografia - Bacharelado (2005) e Licenciatura Plena (2004) pela Universidade Federal de Uberlândia, Minas Gerais, Brasil thaiscardeoli@hotmail.com

\begin{tabular}{|c|} 
Editor Científico: José Edson Lara \\
Organização Comitê Científico \\
Double Blind Review pelo SEER/OJS \\
Recebido em 20.04.2017 \\
Aprovado em 29.06.2017
\end{tabular}




\section{Resumo}

O objetivo deste artigo é analisar trabalhos acadêmicos que relacionam o comportamento do consumidor e as redes sociais, publicados nos principais periódicos brasileiros no período de 2000 a 2015, na área de Administração, Ciências Contábeis e Turismo. Para atender a esse objetivo, realizou-se uma pesquisa bibliográfica a qual possibilitou selecionar artigos relacionados ao tema. Foram encontrados 1.202 trabalhos, dos quais 54 possuíam ligação com o tema. Para a análise dos artigos, utilizou-se um formulário com 11 variáveis e as seguintes comprovações foram obtidas: maior frequência das palavras Facebook e Orkut; $56,9 \%$ dos artigos são de estudos exploratórios e 94,5\%, empíricos; a maioria dos artigos $(75,9 \%)$ foram publicados nos últimos 4 anos; o estado de São Paulo originou 29,5\% dos artigos e o Rio Grande do Sul, 16,8\%; observou-se uma tendência de pesquisas que tratam da relação indivíduo-organização.

Palavras-chave: Redes sociais; Comportamento do consumidor; Orkut; Facebook; Bibliometria.

\section{The research on social networks and consumer behavior in Brazil}

\section{Abstract}

The aim of this paper is to analyze the publication of academic papers relating consumer behavior and social networks, published in the main Brazilian journals from 2000 to 2015, in the area of Administration, Accounting and Tourism. To meet this goal was realized a bibliographic research which allowed select articles related to the topic. They found 1,202 jobs and of these 54 had connection with the theme. For the analysis of articles, we used a form with 11 variables and the following evidences were obtained: (a) greater frequency of Facebook and Orkut words; (b) $56.9 \%$ of the articles are exploratory studies and empirical $94.5 \%$; (c) the vast majority of articles (75.9\%) were published in the last 4 years; (d) highlighting the state of São Paulo, which originated $29.5 \%$ of the authors and Rio Grande do Sul with $16.8 \%$; (e) there was a trend of research dealing with the relationship between individual and organization.

Keywords: Social Network Sites; Consumer's Behavior; Orkut; Facebook; Bibliometrics.

\section{La investigación sobre redes sociales y comportamiento del consumidor en Brasil}

\section{Resumen}

El objetivo de este trabajo es analizar la publicación de trabajos académicos relacionados con el comportamiento del consumidor y las redes sociales, publicado en las principales revistas brasileñas 2000-2015. Para cumplir con este objetivo se realizó una investigación bibliográfica que permitió seleccionar los artículos 
relacionados con el tema. Encontraron 1.202 puestos de trabajo y, de ellos 54 tenían vínculos con el tema. Para el análisis de los artículos, se utilizó un formulario con 11 variables y se obtuvieron los siguientes evidencias: (a) una mayor frecuencia de las palabras de Facebook y Orkut; (b) el 56,9\% de los artículos son estudios exploratorios y empírica $94,5 \%$; (c) la gran mayoría de los artículos $(75,9 \%)$ fueron publicados en los últimos 4 años; (d) hacer hincapié en el estado de Sao Paulo, que se originó el 29,5\% de los autores y Rio Grande do Sul, con un 16,8\%; (e) hubo una tendencia de la investigación trata de la relación entre el individuo y la organización.

Palabras clave: Redes sociales; El comportamiento del consumidor; Orkut; Facebook; Bibliometría.

\section{Introdução}

É notório o avanço da internet nos últimos anos e a sua influência no dia-a-dia das pessoas. De acordo com a União Internacional de Telecomunicações (UIT), haveria cerca de 3 bilhões de usuários de internet no mundo em 2014, sendo dois terços deles oriundos de países em desenvolvimento (Amaral, 2014).

A inclusão digital proporcionou novas perspectivas para o marketing a fim de lidar com clientes e os seus novos hábitos e comportamentos no ambiente virtual. A internet, considerada hoje como um canal de comunicação e comercialização, exige que as empresas busquem melhor visibilidade junto aos consumidores, qualidade que pode ser conquistada por meio do marketing digital (Vaz, 2012). Os sites, blogs, vídeos, fotos, redes sociais, listas de discussão, micro blogs, entre outras ferramentas, podem auxiliar as organizações a alcançarem uma maior notoriedade no mercado.

Caputo (2014) cita o estudo realizado pela UIT, entre setembro de 2013 e fevereiro de 2014, que aponta, pela primeira vez, que mais da metade dos brasileiros foram classificados como usuários de internet em 2014. Destaca-se que apenas $42 \%$ dos brasileiros nunca acessaram a internet. Entre os motivos citados destacam-se falta de habilidade com computador, inexistência de necessidade ou interesse para isso e ausência de condição de pagar pelo acesso.

Ainda conforme o estudo da UIT, $49 \%$ dos domicílios brasileiros têm um computador (Caputo, 2014). A presença de computador é maior nas regiões urbanas, com $53 \%$ de casas com computadores, enquanto nas áreas rurais apenas $21 \%$ das residências contam com esse equipamento. Nas cidades a internet alcança 
25,9 milhões de lares; no campo o número de residências conectadas não passa de 1,3 milhões (Jansen, 2014).

Com relação aos dispositivos móveis, 137 milhões de brasileiros têm um celular. Desses, 31\% usaram internet nesse aparelho nos últimos três meses, o que representa um total de 42,5 milhões de brasileiros (Caputo, 2014).

Em relação ao perfil dos usuários, os jovens continuam a ser os mais conectados: $75 \%$, entre todos os brasileiros de 10 a 15 anos; $77 \%$, entre os de 16 a 24 ; e $66 \%$ entre os de 25 a 34 anos. Já entre os indivíduos na faixa de 35 a 44 anos, $47 \%$ disseram fazer uso da rede. E, apesar da parcela de usuários acima de 45 anos continuar a crescer, saltando de 39\% do segmento em 2012 para $44 \%$ em 2013, essa é a faixa etária com mais brasileiros sem acesso à internet - 45 milhões de pessoas (Jansen, 2014).

Com relação às redes sociais, de acordo com Gomes (2013), o Brasil é o terceiro país com o maior número de pessoas que possuem alguma rede social, atrás apenas dos Estados Unidos, primeiro colocado, e da Índia. No entanto, quando se refere à frequência com que as pessoas usam as redes sociais, o Brasil fica em segundo lugar, perdendo somente para os Estados Unidos.

Segundo Turchi (2012), um importante fator relacionado à internet que catalisou a modificação no comportamento das pessoas nas últimas décadas foi o surgimento das redes sociais. Começou pelo Orkut que, embora tenha sido descontinuado, foi importante para que as pessoas conhecessem as funcionalidades de uma rede social, se adaptassem a ela, reencontrassem amigos e fizessem com que esse tipo de ferramenta tecnológica se tornasse parte das suas vidas. Com isso, de acordo com Turchi (2012), as organizações passaram a se preocupar mais com a compreensão do comportamento dos usuários das redes sociais e os impactos delas nos seus negócios.

De acordo com este contexto, do aumento de pessoas com acesso à internet $\mathrm{e}$ da ampliação da importância das redes sociais no cotidiano das pessoas, o problema de pesquisa desse estudo é: Que tipo de estudos que relacionam as redes sociais e o comportamento do consumidor estão sendo desenvolvidos no Brasil? Sendo assim, o objetivo desta pesquisa é analisar a publicação de trabalhos acadêmicos que relacionam o comportamento do consumidor e as redes sociais, 
publicados nos principais periódicos brasileiros no período de 2000 a 2015, na área de Administração, Ciências Contábeis e Turismo.

A pesquisa se justifica pela importância de estudos sobre o tema, ainda recente e com carência de bibliometrias, que se proponham a verificar o estado da arte sobre $o$ assunto. Justifica-se ainda por contribuir com pesquisas futuras e para a compreensão de conceitos e definições neste campo. A pesquisa bibliométrica, no contexto da produção acadêmica brasileira, é importante porque se torna fonte para revisões de literatura, listando os principais artigos, autores e linhas de pesquisa correntes na academia brasileira.

\section{Referencial teórico}

Para Kleinubing (2010), a bibliometria é uma ferramenta estatística que permite mapear, gerar diferentes indicadores de tratamento, gestão da informação e do conhecimento, especialmente em sistemas de informação e de comunicação científicos, necessários ao planejamento, avaliação e gestão da ciência e da tecnologia de uma determinada comunidade científica ou país.

\subsection{Pesquisas Bibliométricas em Marketing}

O primeiro trabalho brasileiro no campo da Administração a realizar uma análise bibliométrica foi o de Machado-da-Silva, Cunha e Amboni (1990), analisando a perspectiva sobre a pesquisa em organizações. Existem vários outros estudos bibliométricos sobre as diversas áreas que compõem a administração, como Recursos Humanos, Empreendedorismo, entre outros. No contexto de marketing, é possível identificar alguns trabalhos os quais objetivaram a análise da produção brasileira na área. Vieira $(1998,1999,2000,2003)$ realizou uma análise nas publicações brasileiras da disciplina durante a década de 1990, a partir dos anais do EnANPAD, da RAUSP e RAE, com a identificação de 272 artigos.

Ainda em consonância com esses trabalhos supracitados, Mazzon e Hernandez (2013) identificaram que na última década (2000-2009) o interesse maior de pesquisas acadêmicas concentra-se no comportamento do consumidor. Perin, Sampaio, Froemming e Luce (2000) realizaram uma análise acerca das pesquisas 
do tipo survey publicadas na década de 1990 nos anais do EnANPAD, buscando analisar a qualidade das mesmas, considerando o marketing como um todo.

Sobre o marketing de forma geral, podem-se mencionar os trabalhos de Rossi, Bortoli e Castilhos (2012), que efetuaram uma análise bibliométrica da contribuição da área para outras disciplinas, e o estudo de Scharf, Schwingel e Franzon (2013), que realizaram uma pesquisa bibliométrica sobre a produção científica de marketing nos periódicos RAUSP, RAM, READ, CMC e no evento EnANPAD, publicados no período de 2009 e 2010, com um total de 176 artigos analisados. Outro trabalho importante sobre marketing é de Chauvel (1999), que tratou da satisfação do consumidor considerando o pensamento de marketing.

No campo de comportamento do consumidor, Pinto e Lara (2008) realizaram estudo semelhante ao de Perin et al. (2000), porém, além da utilização dos anais do EnANPAD, os autores analisaram também de estudos publicados na RAE, RAC e RAUSP, no período de 1997 a 2006. Ainda sobre comportamento do consumidor, Frio (2013) desenvolveu uma bibliometria sobre esse tema em artigos publicados em periódicos nacionais no período de 2000 a 2011, avaliando um total de 127 artigos disponíveis na internet. Outros estudos a este respeito são o de Troccoli, Gimenez, Reis e Faria (2011), nos quais os autores analisaram as publicações do EnANPAD no período de 2007-2009, e o de Sampaio, Gosling, Fagundes e Veloso (2013) que analisaram as publicações referente ao comportamento do consumidor com relação ao consumo de alimentos orgânicos no período de 1997 a 2011.

Outros autores também desenvolveram pesquisas com o mesmo objetivo na área de marketing: Fagundes, Veiga, Sampaio e Sousa (2012) ponderaram a publicação acadêmica de marketing esportivo no Brasil no período de 1994 a 2011; Lima, Kraemer e Rossi (2013) realizaram uma discussão epistemológica em marketing, analisando artigos, comentários e editoriais no período de 1990-2010 nos 5 principais journals da área segundo o 5-year Impact fator do Journal Citation Reports de 2011; Muylder, Santos, La Falce, Britto e Cançado (2012) realizaram um estudo das principais aplicações de análise discriminante na área de marketing, identificando e analisando 22 artigos publicados na divisão acadêmica de Marketing do EnANPAD que utilizaram esta técnica, no período de 2000 a 2011.

Entre todos esses estudos que usaram a bibliometria para analisar publicações em marketing, nenhum trabalho teve como tema central o estudo do Comportamento 
do Consumidor e Redes Sociais, o que atesta o caráter de ineditismo da pesquisa proposta.

\subsection{Marketing e Marketing Digital}

O marketing, na definição de Kotler e Armstrong (2002), é o processo social e gerencial por meio do qual indivíduos e grupos obtêm aquilo de que necessitam e desejam. Cobra (1993) menciona em seu trabalho que algumas pessoas relacionam marketing apenas com propaganda ou com vendas, mas para ele o marketing é mais do que isso, uma vez que integra forças para colocar o produto certo no local certo, sendo mais do que um exercício de negociação entre produtores e distribuidores, uma vez que todos na empresa devem refletir e agir sob a ótica do marketing.

Contudo, nota-se nos últimos anos uma mudança de como chegar até o consumidor e apresentar o produto e/ou serviço. Com o advento da internet, e como cada vez mais as pessoas estão conectadas uma com as outras por meio dessa mídia, surgiu a necessidade de o marketing desenvolver estratégias voltadas para o ambiente virtual da internet. Nesse sentido, Laudon e Laudon (2004) consideram a internet uma ferramenta poderosa para vendas e marketing, pois fornece capacidades para a personalização e interação com clientes que dificilmente são encontradas em outros canais. As organizações podem manter diálogos continuados com clientes, usando e-mail, bate-papo e grupos de discussão eletrônica, para solidificar seus relacionamentos com eles.

Com a evolução da tecnologia da informação e da comunicação, especialmente a internet, o Marketing evoluiu para o chamado Marketing eletrônico, e-Marketing ou Marketing digital, conceito que expressa o conjunto de ações de Marketing intermediadas por canais eletrônicos como a internet, em que o cliente controla a quantidade e o tipo da informação recebida (Limeira, 2003, p. 9).

Dessa forma, segundo Torres (2009, p. 7), "o marketing digital está se tornando cada dia mais importante para os negócios e para as empresas". Não é uma questão de tecnologia, mas uma mudança no comportamento do consumidor, que está utilizando cada vez mais a internet como meio de comunicação, relacionamento e entretenimento. Portanto, o marketing digital pode ser uma ferramenta utilizada por empresas em ações de comunicação, quer seja telefonia celular, informática ou outros meios digitais existentes, com a finalidade de 
conquistar e se relacionar com novos clientes. $O$ autor ainda considera que a publicidade online oferece diversas ferramentas para divulgar produtos ou serviços, como redes sociais, sites de pesquisa, portais de conteúdo, blogs, entre outros.

Reforça-se que este trabalho busca analisar o comportamento do consumidor nas redes sociais. Para tanto, será considerado apenas o marketing nas mídias sociais - tópico que será tratado na sequência -que permite que a marca amplie seu relacionamento com o cliente e possibilita que ela possa divulgar suas ações e informações, além de incentivar o compartilhamento de conteúdo e contribuir para a divulgação da marca. A vantagem de alta visibilidade e da possibilidade de construção de relacionamentos nas redes sociais, aliado à grande interatividade proporcionada por essa ferramenta, é o que as tornam atraentes para as organizações (Lemes \& Ghisleni, 2013).

\subsection{Redes Sociais}

As redes sociais, do ponto de vista conceitual, são definidas e classificadas de diversas formas e com critérios distintos, o que torna a conceitualização do termo algo complexo. Recuero (2009, p. 24) caracteriza rede social como

um conjunto de dois elementos: atores (pessoas, instituições ou grupos, os nós da rede) e suas conexões (interações ou laços sociais) [...] Uma rede, assim, é uma metáfora para observar os padrões de conexão de um grupo social, a partir das conexões estabelecidas entre os diversos autores. A abordagem da rede tem, assim, seu foco na estrutura social, onde não é possível isolar os autores sociais e nem suas conexões.

Marteleto e Silva (2004) ressaltam a importância da análise das redes sociais para que se possa compreender o processo de acesso à informação, bem como o desenvolvimento da sociedade do ponto de vista econômico e social. Dessa forma, considera-se que redes sociais são ambientes cujo foco é reunir pessoas, os chamados membros, que, uma vez inscritos, podem expor seu perfil com dados como fatos pessoais, textos, mensagens e vídeos - além de interagir com outros membros, criando listas de amigos e comunidades (Telles, 2011).

Para Kimura, Basso e Martin (2008), as redes sociais são uma representação das relações e interações entre indivíduos de um grupo e têm um papel importante como meio de propagação de informações, ideias e influências. Essas redes afetam 
a estrutura da comunicação, isso porque antes as redes sociais tinham mais controle sobre seus canais de comunicação, mas hoje elas interagem em espaços não mais sob seu controle (Hercheui, 2011).

Nas estratégias de marketing voltadas para as redes sociais é essencial que se tenha um conjunto de ações que sigam um plano coerente e consistente. Informações cruciais, como o comportamento de consumo dos usuários, a identificação do público-alvo, a região em que esses usuários se encontram, as palavras-chave mais digitadas por eles, entre outras, referem-se a algumas das informações que os gestores de marketing devem buscar (Vaz, 2008).

No ano de 2004, as redes sociais se popularizaram, principalmente com a criação do Orkut e do Facebook. Em 2006, o Facebook deixou de ter seu acesso restrito e passou a ser liberado para qualquer usuário com mais de 13 anos; nesse mesmo período surgiu o Twitter. Em 2009, com a popularização do Facebook e Twitter, várias empresas aderiram às duas redes sociais (Guimarães, 2011).

Destaca-se que o avanço da tecnologia propiciou novos hábitos de consumo e, principalmente, alterou a maneira como os consumidores acessam a informação (Bertoncello \& Crescitelli, 2009). Nesse contexto, com as redes sociais as pessoas não apenas compartilham suas opiniões, como são simultaneamente influenciadas pelas interações que estabelecem. Essa livre expressão de ideias e experiências pode fornecer às organizações informações valiosas para o desenvolvimento das suas estratégias de marketing (Stanley, 2009).

As necessidades e desejos dos consumidores ficam expostos por meio de compartilhamentos, "curtidas" e postagens. Não apenas isso, mas, como avaliam Maya e Otero (2002), o surgimento de comunidades virtuais agrega pessoas com interesses comuns e cria efeitos que alteram as relações de consumo, o que gera a necessidade das organizações se adaptarem a essas mudanças.

\subsection{Comportamento do Consumidor}

Blessa (2006) afirma que o comportamento de consumo é definido como as ações que impactam na procura, compra, uso e avaliação de produtos ou serviços. Esse comportamento pode ser descrito como uma atividade física, mental e emocional realizada na seleção, compra e uso de produtos ou serviços para satisfação de necessidades e desejos. 
Samara e Morsch (2005) afirmam que compreender o consumidor é uma função essencial do marketing, para que ele possa cumprir plenamente seus objetivos no desenvolvimento, na produção e na colocação no mercado de bens e serviços apropriados e capazes de satisfazer às necessidades e desejos dos consumidores, além de auxiliar o desenvolvimento de estratégias de segmentação e posicionamento.

A mudança nos hábitos de consumo e das formas de relacionamento entre o varejo e os consumidores normalmente é motivada pela adoção de tecnologias digitais, como a internet e as redes sociais, formando um consumidor digital, multicanal e global, que tem perspectivas internacionais para o seu consumo. Normalmente, esse consumidor é mais maduro, crítico e racional, e a internet se caracteriza por exercer grande influência na escolha de produtos e serviços à medida que permite comparações e informações sobre características, preços, condições, serviços, garantias, formas de uso, entre outras, que impactem em uma decisão de compra (Souza, 2010).

Stanley (2009) considera que parte do comportamento e das expectativas das pessoas estão sendo moldados pela web, e o crescente desejo de compartilhar opiniões, experiências e aspirações possibilita às organizações uma oportunidade única de captar, medir e conectar dados para depois utilizá-los no desenvolvimento de ações de marketing na internet e, especificamente, nas redes sociais.

De acordo com Kotler e Armstrong (1995), o comportamento de compra é influenciado por, principalmente, fatores culturais, sociais, pessoais e psicológicos. Os fatores culturais são aqueles advindos do ambiente em que a pessoa está inserida e corresponde aos valores e às percepções que são adquiridas desde a infância. Os fatores sociais relacionam-se com os grupos dos quais as pessoas fazem parte dentro da sociedade e a forma como interagem com os indivíduos desses grupos. As redes sociais virtuais, foco desse estudo, têm impactado significativamente neste fator, pois as pessoas formam grupos com ideais, gostos e opiniões semelhantes.

Outro fator que influencia o comportamento de compra são os pessoais, que estão relacionados com a forma como a pessoa vive na sociedade, seu ciclo de vida e preferências. O último fator é o psicológico, que representa todas as dimensões que estão diretamente ligadas com a satisfação. Inserem-se nesse aspecto as 
características relacionadas com a motivação, a percepção, a experiência e tudo que a pessoa julga ser importante para sua vida (Kotler \& Armstrong, 1995).

\section{Aspectos metodológicos}

Neste trabalho desenvolve-se uma pesquisa bibliográfica (Vergara, 2009), que analisou a publicação acadêmica brasileira sobre o tema comportamento do consumidor e redes sociais, analisando-se os artigos publicados no período de 2000 a 2015 em periódicos brasileiros. A pesquisa foi empreendida nos meses de março a abril de 2016.

Quanto aos fins, a pesquisa se classifica como descritiva, porque visa apresentar, classificar e analisar os artigos selecionados, ou seja, expõe características de determinado fenômeno (Vergara, 2009).

Utilizou-se a técnica de pesquisa documental, na qual "a fonte de coleta de dados está restrita a documentos, escritos ou não, constituindo o que se denomina de fontes primárias" (Marconi \& Lakatos, 2007, p. 176), visto que foram analisados artigos de comportamento do consumidor e redes sociais publicados no Brasil no período de 2000 a 2015.

Flick (2004) aponta a importância de trabalhos acadêmicos que busquem analisar e avaliar a publicação de outros estudos anteriormente realizados, considerando que esse tipo de pesquisa pode incrementar e aperfeiçoar a publicação do tema objeto de estudo. Assim, a bibliometria pode ser considerada como útil para o tratamento técnico e para a gestão da informação e do conhecimento produzidos em campos científicos determinados (Guedes \& Borshiver, 2005).

Como forma de nortear pesquisas bibliométricas, algumas normas foram criadas, como: (a) norma de Bradford (produtividade de periódicos); (b) norma de Lotka (produtividade científica de autores); e (c) norma de Zipf (frequência de palavras). A norma de Bradford, relacionada à dispersão da literatura periódica científica, indica que quando pesquisadores escrevem novos artigos sobre um determinado assunto e o submeterem a uma pequena seleção de periódicos apropriados, uma vez aceitos, vários outros pesquisadores sobre o mesmo assunto serão atraídos para publicar nesse periódico. Paralelamente, outros periódicos tendem a publicar pela primeira vez artigos sobre o assunto e, se a temática 
continuar a se desenvolver, surgirão núcleos de periódicos (Guedes \& Borshiver, 2005).

Já a norma de Lotka está relacionada à produtividade dos autores. Essa regra leva em consideração que "alguns pesquisadores publicam muito e muitos publicam pouco" (Voos, 1974 como citado em Guedes \& Borschiver, 2005, p. 5). Complementarmente, na regra de Zipf é possível estimar que um pequeno grupo de palavras ocorre muitas vezes, e um grande número de palavras é de pequena frequência de ocorrência. Esse estudo leva em consideração as frequências de ocorrência das palavras em um determinado texto científico, além da região de concentração de termos de indexação, ou palavras-chave (Guedes \& Borschiver, 2005). Dessa forma, este trabalho apresenta mais características relacionadas à norma de Bradford.

O presente estudo considerou os artigos publicados em periódicos brasileiros melhores classificados - estratos A1, A2, B1 e B2 - no sistema WebQualis na área de Administração, Ciências Contábeis e Turismo (CAPES, 2012). A escolha por essas fontes deve-se ao fato de as mesmas serem publicadas no Brasil e de serem importantes veículos de divulgação de pesquisas acadêmicas desenvolvidas no País. Ademais, também se optou por selecionar fontes de publicação que priorizam submissões de trabalhos referentes à área de Administração e que aceitam pesquisas sobre marketing.

O procedimento de seleção dos artigos considerados para avaliação referentes ao comportamento do consumidor e redes sociais envolveu a busca no banco de dados dos periódicos que participaram da amostra. Para essa busca, selecionaramse todos os artigos que apresentavam como título, palavras-chave ou assunto as seguintes palavras ou expressões: "redes sociais"; "mídias sociais"; "Facebook"; "Orkut"; "Instagram" e "Twitter" - essas redes sociais foram escolhidas por serem as que possuem maior número de usuários. Nessa primeira fase foram encontrados 1.202 trabalhos.

Posteriormente, esses trabalhos foram avaliados para se analisar se os mesmos tratavam, realmente, de redes sociais virtuais e comportamento do consumidor. Nessa segunda fase de seleção foram descartados 1.148 trabalhos que não tratavam dos temas estudados, resultando assim em 54 artigos para serem analisados. 
Para a análise dos 54 artigos foi elaborado um formulário que envolveu as seguintes variáveis, adaptadas de Botelho e Macera (2001), Perin et al. (2000), Vieira (1998; 2000), Pinto e Lara (2007) e Fagundes et al. (2012):

a) Dados do artigo - local de publicação, ano de publicação e quantidade de autores.

b) Dados dos autores - gênero, titulação, instituição de vínculo e estado da instituição.

c) Tipo de estudo - empírico ou teórico.

d) Abordagem da pesquisa - qualitativa ou quantitativa.

e) Natureza da pesquisa - exploratória, descritiva, causal ou outra.

f) Metodologia de pesquisa - survey, experimentos, estudo de caso ou outra.

g) Tipos de dados coletados - primário ou secundário.

h) Abrangência do estudo - longitudinal ou transversal.

i) Instrumento de coleta de dados utilizado.

j) Segmento de consumidores pesquisados.

k) Forma de relacionamento/interação entre os agentes (organização-indivíduos ou vice-versa; triangulações organização-indivíduo-organização ou indivíduoorganização-indivíduo).

Após essa avaliação, os dados coletados foram tabulados e analisados utilizando-se o software SPSS 20.

\section{Análise dos artigos}

Entre os 54 artigos analisados, o periódico que mais apresentou artigos relacionados com os temas pesquisados foi a Revista Brasileira de Marketing (ReMark), com 6 trabalhos. Em seguida, com 4 artigos, estão a Revista Pensamento Contemporâneo em Administração (RPCA) e a Contemporânea - Revista de Comunicação e Cultura. Também apresentaram quantidade relevante de trabalhos os periódicos Organizações em Contexto, Revista Brasileira de Pesquisa em Turismo (RBTUR), Revista de Administração Contemporânea (RAC) e Revista Gestão \& Tecnologia, todos com 3 publicações. Esses sete periódicos, somados, representam $48,1 \%$ do total de artigos encontrados, conforme observa-se na Tabela 1. 


\section{Tabela 1}

Distribuição dos artigos em relação ao periódico

\begin{tabular}{|c|c|c|}
\hline Título Periódico & Frequência & $\%$ \\
\hline Revista Brasileira de Marketing - REMark & 6 & $11,1 \%$ \\
\hline Contemporânea - Revista de Comunicação e Cultura & 4 & $7,4 \%$ \\
\hline Revista Pensamento Contemporâneo em Administração - RPCA & 4 & $7,4 \%$ \\
\hline Organizações em Contexto & 3 & $5,6 \%$ \\
\hline Revista Brasileira de Pesquisa em Turismo - RBTUR & 3 & $5,6 \%$ \\
\hline Revista de Administração Contemporânea - RAC & 3 & $5,6 \%$ \\
\hline Revista Gestão \& Tecnologia & 3 & $5,6 \%$ \\
\hline $\begin{array}{ll}\text { Título Periódico } \\
\end{array}$ & Frequência & $\%$ \\
\hline Caderno Virtual de Turismo & 2 & $3,7 \%$ \\
\hline Perspectivas em Ciência da Informação & 2 & $3,7 \%$ \\
\hline Revista de Administração da UNIMEP & 2 & $3,7 \%$ \\
\hline Revista de Administração, Contabilidade e Economia & 2 & $3,7 \%$ \\
\hline Análise (Revista de Administração da PUCRS) & 1 & $1,9 \%$ \\
\hline Revista Brasileira De Ciências Sociais & 1 & $1,9 \%$ \\
\hline Revista Brasileira de Ecoturismo & 1 & $1,9 \%$ \\
\hline Revista Brasileira de Gestão de Negócios & 1 & $1,9 \%$ \\
\hline Revista Brasileira de Inovação & 1 & $1,9 \%$ \\
\hline Revista de administração & 1 & $1,9 \%$ \\
\hline Revista de Administração da UFSM & 1 & $1,9 \%$ \\
\hline Revista de Administração de Empresas - ERA & 1 & $1,9 \%$ \\
\hline Revista de Administração e Contabilidade da UNISINOS & 1 & $1,9 \%$ \\
\hline Revista de Administração e Inovação & 1 & $1,9 \%$ \\
\hline Revista de Administração Mackenzie & 1 & $1,9 \%$ \\
\hline Revista de Negócio & 1 & $1,9 \%$ \\
\hline Revista Digital de Biblioteconomia e Ciência da Informação & 1 & $1,9 \%$ \\
\hline Revista Economia \& Gestão - E\&G & 1 & $1,9 \%$ \\
\hline Revista Eletrônica de Sistemas de Informação & 1 & $1,9 \%$ \\
\hline Revista Eletrônica Sistemas \& Gestão & 1 & $1,9 \%$ \\
\hline Revista G.U.A.L. & 1 & $1,9 \%$ \\
\hline Revista Produção On Line & 1 & $1,9 \%$ \\
\hline Sistemas e Gestão Revista Eletrônica & 1 & $1,9 \%$ \\
\hline TransInformação & 1 & $1,9 \%$ \\
\hline Total & 54 & \\
\hline
\end{tabular}

Nota. Fonte: Elaborada pelos autores.

A Figura 1 apresenta a quantidade de publicações sobre o tema pesquisado no período analisado -2000 a 2015 . Nota-se que houve um aumento significativo de produções do ano de 2011 para o ano de 2012, evolução de 3 para 10, e esse número de publicações persistiu, sendo que no ano de 2014 obtiveram-se 15 trabalhos publicados que envolviam o comportamento dos consumidores e redes sociais. Todavia, em 2015 houve uma diminuição no número de publicações de artigos sobre essa temática. Apesar da pesquisa ter analisado trabalhos de 2000 até 2015, a grande concentração de artigos a respeito do tema foi a partir de 2011, 
sendo esse período responsável por $81,5 \%$ do total. Acredita-se que esse fato se deve à maior importância que o tema vem conquistando nesses últimos anos.

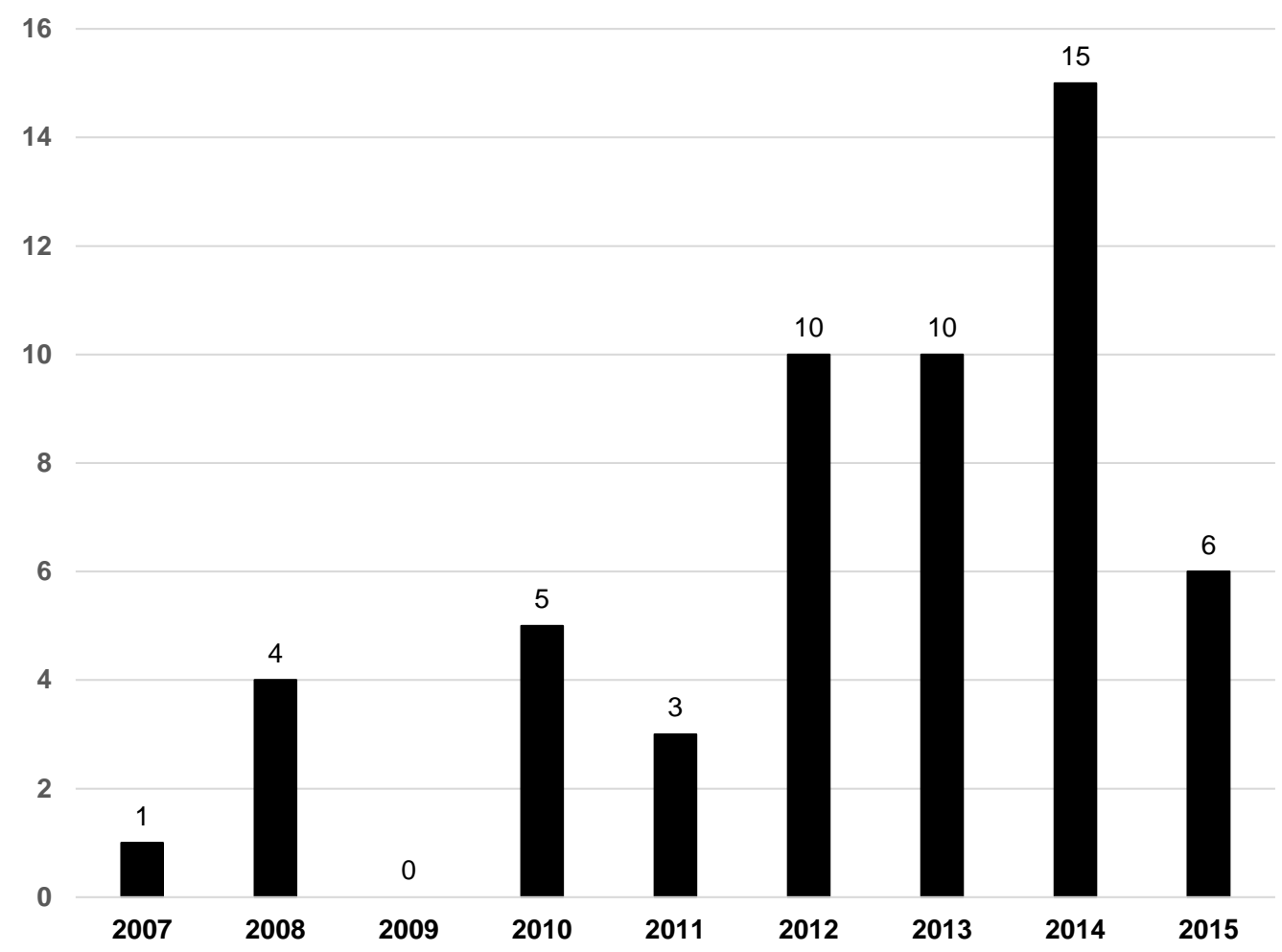

Figura 1

Artigos publicados no Brasil entre 2010 e 2015 sobre redes sociais e comportamento do consumidor

Nota: Entre 2000 e 2006 não foram encontrados nenhum artigo publicado sobre 0 assunto. Fonte: Elaborado pelos autores.

De acordo com os dados dos artigos analisados, do total de autores, $62 \%$ são homens. Considerando a titulação dos autores na data de publicação do artigo, 68 possuíam doutorado, 45 mestrado e 33 graduação. Todavia, não foi possível encontrar a titulação de 3 autores. A média de autores por artigo é 2,7.

$\mathrm{Na}$ Tabela 2 estão listadas as instituições de origem dos autores. Destaque para a Universidade Federal do Rio Grande do Sul, com 16 autores (10,7\%), a Universidade Presbiteriana Mackenzie, com 10 pesquisadores (6,7\%), além da Fundação Getúlio Vargas e da Universidade Federal de Minas Gerais, ambas com 9 autores $(6,0 \%)$. 

no Brasil

Tabela 2

Distribuição dos autores dos artigos em relação a sua instituição de origem

\begin{tabular}{|c|c|c|}
\hline Instituição de Ensino & Frequência & $\%$ \\
\hline Universidade Federal do Rio Grande do Sul & 16 & $10,7 \%$ \\
\hline Universidade Presbiteriana Mackenzie & 10 & $6,7 \%$ \\
\hline Fundação Getúlio Vargas de São Paulo & 9 & $6,0 \%$ \\
\hline Universidade Federal de Minas Gerais & 9 & $6,0 \%$ \\
\hline Pontifícia Universidade Católica do Rio de Janeiro & 8 & $5,4 \%$ \\
\hline Universidade Federal do Paraná & 8 & $5,4 \%$ \\
\hline Universidade do Estado de Santa Catarina & 7 & $4,7 \%$ \\
\hline Universidade Federal de Santa Maria & 5 & $3,4 \%$ \\
\hline Instituição de Ensino & Frequência & $\%$ \\
\hline Universidade Federal Rural de Pernambuco & 5 & $3,4 \%$ \\
\hline Escola Superior de Propaganda e Marketing & 4 & $2,7 \%$ \\
\hline Universidade de São Paulo & 4 & $2,7 \%$ \\
\hline Universidade Estadual Júlio Mesquita Filho & 4 & $2,7 \%$ \\
\hline Pontifícia Universidade Católica de Minas Gerais & 3 & $2,0 \%$ \\
\hline Pontifícia Universidade Católica de São Paulo & 3 & $2,0 \%$ \\
\hline Universidade do Amazonas & 3 & $2,0 \%$ \\
\hline Universidade do Sul de Santa Catarina & 3 & $2,0 \%$ \\
\hline Universidade do Vale do Itajaí & 3 & $2,0 \%$ \\
\hline Universidade Federal da Bahia & 3 & $2,0 \%$ \\
\hline Universidade Federal de São Carlos & 3 & $2,0 \%$ \\
\hline Universidade Federal Fluminense & 3 & $2,0 \%$ \\
\hline Vienna University of Economics and Business & 3 & $2,0 \%$ \\
\hline Faculdade Campo Limpo Paulista & 2 & $1,3 \%$ \\
\hline Fundação Mineira de Educação e Cultura - FUMEC & 2 & $1,3 \%$ \\
\hline Instituto Superior de Entre Douro e Vouga & 2 & $1,3 \%$ \\
\hline Pontifícia Universidade Católica do Rio Grande do Sul & 2 & $1,3 \%$ \\
\hline Universidade Autônoma de Barcelona & 2 & $1,3 \%$ \\
\hline Universidade Estadual do Ceará & 2 & $1,3 \%$ \\
\hline Universidade Estadual Paulista & 2 & $1,3 \%$ \\
\hline Universidade Federal de Santa Catarina & 2 & $1,3 \%$ \\
\hline Universidade Federal do Rio de Janeiro & 2 & $1,3 \%$ \\
\hline Faculdade de Sumaré & 1 & $0,7 \%$ \\
\hline Instituto Federal de São Paulo & 1 & $0,7 \%$ \\
\hline Pontifícia Universidade Católica do Paraná & 1 & $0,7 \%$ \\
\hline Universidade Católica do Rio Grande do Sul & 1 & $0,7 \%$ \\
\hline Universidade do Oeste de Santa Catarina & 1 & $0,7 \%$ \\
\hline Universidade Federal de Alagoas & 1 & $0,7 \%$ \\
\hline Universidade Federal de Lavras & 1 & $0,7 \%$ \\
\hline Universidade Federal de Ouro Preto & 1 & $0,7 \%$ \\
\hline Universidade Federal de Sergipe & 1 & $0,7 \%$ \\
\hline Universidade Federal de Uberlândia & 1 & $0,7 \%$ \\
\hline Universidade Federal do Ceará & 1 & $0,7 \%$ \\
\hline Universidade Federal do Pampa & 1 & $0,7 \%$ \\
\hline Universidade Metodista de São Paulo & 1 & $0,7 \%$ \\
\hline Université Pierre Mendès & 1 & $0,7 \%$ \\
\hline Université René Descartes & 1 & $0,7 \%$ \\
\hline Total & 149 & \\
\hline
\end{tabular}

Nota. Fonte: Elaborada pelos autores. 
Considerando-se o estado de origem das instituições, São Paulo tem 47 dos 149 autores $(29,5 \%)$ e o Rio Grande do Sul 25 autores (16,8\%). É importante destacar que dentre os artigos publicados nos periódicos brasileiros analisados 4 são de autores com origem em outros países: Portugal, França, Áustria e Espanha.

Com relação às características dos artigos na área de redes sociais e comportamento do consumidor, em sua maioria $(94,5 \%)$ foram realizados estudos empíricos e apenas 4,5\% (3 trabalhos) são exclusivamente teóricos. Observa-se ainda que em 25 trabalhos (46,3\%) foram realizadas pesquisas qualitativas, 20 $(37,0 \%)$ estudos quantitativos e apenas $9(16,7 \%)$ foram pesquisas que abordaram os dois métodos - qualitativa-quantitativa. Em relação à natureza das pesquisas, $56,9 \%$ foram de origem exploratória e $37,5 \%$ descritivas - Tabela 3 .

\section{Tabela 3}

Natureza da pesquisa abordada nos artigos analisados

\begin{tabular}{lcc}
\hline \multicolumn{1}{c}{ Natureza da pesquisa } & Frequência & $\%$ \\
\hline Exploratória & 41 & $56,9 \%$ \\
Descritiva & 27 & $37,5 \%$ \\
Comparativa & 2 & $2,8 \%$ \\
Interpretativa & 2 & $2,8 \%$ \\
\hline Total & $\mathbf{7 2}^{\boldsymbol{*}}$ &
\end{tabular}

Nota. A soma ultrapassa o total de artigos pesquisados devido ao fato de alguns trabalhos terem sido classificados como pertencentes a mais de uma categoria analisada.

Fonte: Elaborada pelos autores.

Com relação às metodologias de pesquisa empreendidas nos artigos, o estudo de caso predominou, com $37,0 \%$, seguido do uso de survey $(24,1 \%)$, conforme a Figura 2.

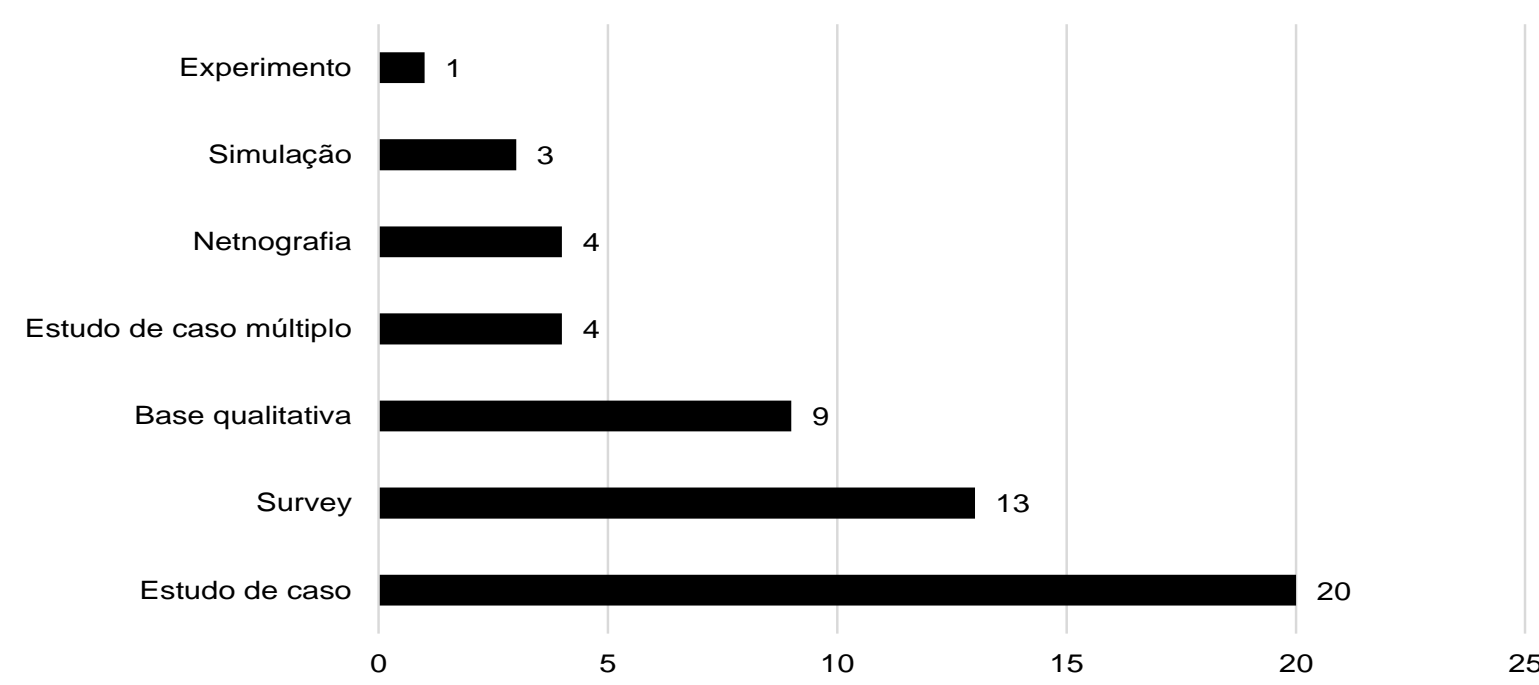

Figura 2

Metodologia de Pesquisa usada nos artigos

Fonte: Elaborado pelos autores. 
Referente ao tipo de dado que os autores dos artigos utilizaram, 70,4\% (38 artigos) usaram dados primários, 10 (18,5\%) dados secundários e 6 (11,1\%) optaram por usar ambos os tipos. Ademais, constatou-se que todos os artigos realizaram estudos transversais.

Analisando-se 0 instrumento de coleta de dados utilizado nas pesquisas, observou-se que o mais usado foi o levantamento em site $(37,9 \%)$. Questionário eletrônico foi o segundo instrumento mais utilizado, seguido de entrevistas semiestruturadas. Somando estes três tem-se $69,7 \%$ dos instrumentos usados Tabela 4.

\section{Tabela 4}

Instrumento de coleta de dados usados nos artigos analisados

\begin{tabular}{lcc}
\hline \multicolumn{1}{c}{ Instrumento de coleta de dados } & Frequência & $\%$ \\
\hline Levantamento em sites & 25 & $37,9 \%$ \\
Questionário eletrônico & 12 & $18,2 \%$ \\
Entrevistas semi-estruturadas & 9 & $13,6 \%$ \\
Instrumento de outra pesquisa - simulação & 4 & $6,1 \%$ \\
Pesquisa bibliográfica & 4 & $6,1 \%$ \\
Observação participante & 4 & $6,1 \%$ \\
Pesquisa documental & 3 & $4,5 \%$ \\
Entrevista em profundidade & 2 & $3,0 \%$ \\
Entrevistas estruturadas & 2 & $3,0 \%$ \\
Grupo Focal & 1 & $1,5 \%$ \\
\hline Total & $66^{\star}$ & \\
\hline
\end{tabular}

Nota. A soma ultrapassa o total de artigos pesquisados devido ao fato de alguns trabalhos terem sido classificados como pertencentes a mais de uma categoria analisada.

Fonte: Elaborada pelos autores.

Também buscou-se verificar a estrutura dos artigos analisados, conforme Tabela 5. Verifica-se que os artigos apresentam, na sua maioria, as seguintes características: teorias e conceitos de base $(98,1 \%)$, objetivos da pesquisa $(98,1 \%)$, questões de pesquisa $(94,4 \%)$, tipos de dados coletados $(85,2 \%)$ e a forma de coleta de dados $(92,6 \%)$.

\section{Tabela 5}

Itens que constam na estrutura dos artigos analisados

\begin{tabular}{lcccc}
\hline \multirow{2}{*}{\multicolumn{1}{c}{ Itens }} & \multicolumn{2}{c}{ Sim } & \multicolumn{2}{c}{ Não } \\
\cline { 2 - 5 } & Freq. & $\%$ & Freq. & $\%$ \\
\hline Teorias e conceitos de base & 53 & $98,1 \%$ & 1 & $1,9 \%$ \\
Objetivos da pesquisa & 53 & $98,1 \%$ & 1 & $1,9 \%$ \\
Questões de pesquisa & 51 & $94,4 \%$ & 3 & $5,6 \%$ \\
Hipóteses ou pressupostos de base & 15 & $27,8 \%$ & 39 & $72,2 \%$ \\
\hline Tipos de dados coletados & 46 & $85,2 \%$ & 8 & $14,8 \%$ \\
Forma de coleta de dados & 50 & $92,6 \%$ & 4 & $7,4 \%$ \\
\hline
\end{tabular}




\begin{tabular}{lllll}
\hline Tipo de amostra & 31 & $57,4 \%$ & 23 & $42,6 \%$ \\
\hline Limites do estudo & 36 & $66,7 \%$ & 18 & $33,3 \%$ \\
$\begin{array}{l}\text { Recomendações para pesquisas } \\
\text { futuras }\end{array}$ & 29 & $53,7 \%$ & 25 & $46,3 \%$ \\
$\begin{array}{l}\text { Recomendações para aplicação } \\
\text { prática }\end{array}$ & 20 & $37,0 \%$ & 34 & $63,0 \%$ \\
\hline
\end{tabular}

Nota. Fonte: Elaborada pelos autores.

Referente ao tipo de amostra, recomendações para futuras pesquisas e limites do estudo, mais da metade dos artigos apresentaram tais itens. No entanto, os itens hipóteses ou pressupostos de base (presente em 27,8\% dos artigos) e recomendações para aplicação prática (37\%) não foram encontrados na maioria dos trabalhos.

Ademais, analisou-se o tipo de interação pesquisado nos artigos, tendo-se como foco organizações e indivíduos e a direção dessa relação - as possibilidades eram que a análise fosse apenas da organização, apenas do indivíduo, da organização para o indivíduo e do indivíduo para a organização. Os resultados apontam que a maioria das pesquisas $(53,7 \%)$ apresentam relação do indivíduo para a organização, o que sugere que os autores estudaram como as redes sociais estão servindo para que as pessoas mostrem seus pensamentos e atitudes em relação às organizações. Do total, 12 artigos não apresentaram relação de interação, sendo 10 focados somente no indivíduo e 2 exclusivos na organização, o que representa $25,9 \%$ do total analisado. Referente à relação de troca entre organização-indivíduo e indivíduo-organização, apenas 4 publicações $(7,4 \%)$ apresentaram essa relação, o que significa que esses artigos analisaram não somente as atitudes dos consumidores com relação às empresas, mas também o que as empresas fazem com relação aos consumidores.

Ainda poder-se-ia classificar essa interação como uma triangulação do tipo consumidor-organização-consumidor, ou seja, o consumidor usa as redes sociais para manifestar sua opinião e a empresa apresenta um retorno, ou mesmo organização-consumidor-organização. No entanto, conforme apresentado na Tabela 6, nenhum artigo estudou triangulações desses dois últimos tipos. 


\section{Tabela 6}

Tipos de interação entre organização e indivíduo

\begin{tabular}{lcc}
\hline Tipo de interação entre organização e indivíduo & Frequência & $\%$ \\
\hline Indivíduo-organização & 29 & $53,7 \%$ \\
Indivíduo & 10 & $18,5 \%$ \\
Organização-indivíduo & 9 & $16,7 \%$ \\
Indivíduo-organização e organização-Indivíduo & 4 & $7,4 \%$ \\
Organização & 2 & $3,7 \%$ \\
Total & $\mathbf{5 4}$ & \\
\hline
\end{tabular}

Nota. Fonte: Elaborada pelos autores.

Por fim, buscou-se identificar o segmento de consumo pesquisado nos artigos analisados. Nesse aspecto, não foi possível identificar um padrão nas pesquisas. Usuários de redes sociais, de forma geral, foi o segmento que apresentou maior frequência nos artigos avaliados (14). Em seguida, com 3 publicações, aparecem as pesquisas com alunos de graduação, consumidores de cosméticos e análise de usuários de sites de empresas. Os demais artigos tratam de diversos assuntos, não sendo possível agrupá-los em um mesmo segmento, sendo que os temas encontrados foram os seguintes: comportamento de adolescentes, consumidores de bebidas alcoólicas, turismo, pessoas se preparando para o casamento, uso de celulares, avaliação de hotéis, fãs de voleibol, empresas incubadoras, consumidores de comércio eletrônico, turistas usuários de telefonia móvel, pessoas resistentes ao celular, pessoas resistentes a marcas específicas, consumidores de moda, consumidores que fazem publicidade, usuários de hotéis de pequeno porte, usuários de serviços turísticos em eventos, serviços de hotelaria, franqueados, eleitores e ativistas virtuais. No entanto, 9 artigos não deixaram especificado qual o segmento de consumidor se estava estudando.

Buscando apresentar uma síntese dos resultados encontrados nesta pesquisa, a Figura 3 apresenta as características que predominaram nos artigos analisados neste estudo.

\begin{tabular}{|l|l|}
\hline \multicolumn{1}{c}{ Variável } & \multicolumn{1}{c}{ Maior Frequência } \\
\hline Ano de maior publicação & 2014 \\
\hline Gênero predominante dos pesquisadores & Homens \\
\hline Titulação predominante dos pesquisadores & Doutorado \\
\hline Instituições de origem da maioria dos autores & $\begin{array}{l}\text { Universidade Federal do Rio Grande do } \\
\text { Sul }\end{array}$ \\
\hline Estado de origem da maioria das IES dos autores & São Paulo \\
\hline Principal tipo de estudos desenvolvidos & Estudos empíricos \\
\hline Principal natureza da pesquisa dos artigos & Exploratória \\
\hline
\end{tabular}




\begin{tabular}{|l|l|}
\hline Método de pesquisa mais utilizado & Estudo de Caso \\
\hline \multicolumn{1}{|l|}{ Tipo de dado mais coletado } & Primários \\
\hline Instrumento de coleta de dados mais utilizado & Levantamento em site \\
\hline Tipo de interação entre organização e indivíduo & \multicolumn{1}{|c|}{ Indivíduo-organização } \\
\hline Segmento de consumidor mais pesquisado & Usuários de redes sociais de forma geral \\
\hline
\end{tabular}

Figura 3

Variáveis analisadas e maior frequência

Fonte: Elaborada pelos autores.

\section{Considerações finais}

Esta pesquisa bibliométrica visou analisar a publicação de artigos acadêmicos sobre redes sociais e comportamento do consumidor, publicados nos principais periódicos brasileiros (classificados como A1, A2, B1 ou B2 no sistema WebQualis) entre os anos de 2000 e 2015, na área de Administração, Ciências Contábeis e Turismo. A partir da análise destes trabalhos podem-se tecer considerações sobre as características gerais dos mesmos, especificidades e, assim, subsidiar futuras pesquisas sobre o tema.

Entre os trabalhos publicados, foram encontrados um total de 1.202 artigos que apresentavam uma ou mais das palavras-chaves pesquisadas (redes sociais, mídias sociais, Facebook, Orkut, Instagram e Twitter). Destes, apenas 54 tratavam especificamente do tema de pesquisa deste trabalho, o que corresponde a $4,5 \%$ do total inicialmente selecionado. Isto se justifica por 'redes sociais' ser um conceito amplo e abordado de formas distintas nas revistas da área de Administração, Ciências Contábeis e Turismo, como, por exemplo, redes de cooperação local, redes de saúde, redes para reivindicar os direitos das minorias, entre outras. Outro ponto a se observar é que a maioria dos artigos descartados trata de redes inter e intraorganizacionais, ou seja, aquelas que promovem a interação entre organizações ou entre os setores de uma organização.

Ao se pesquisarem os artigos para este estudo bibliométrico, percebeu-se que houve uma maior frequência das palavras Facebook e Orkut. Acredita-se que isso relaciona-se ao fato de o Orkut ter sido a primeira rede social a ganhar expressividade no Brasil, em meados da década de 2000, e à popularização do Facebook entre os brasileiros no final da mesma década. O Facebook pode ainda render muitos estudos na área, tendo em vista que essa rede social encontra-se ativa e com grande número de usuários. 
Por outro lado, a pouca frequência da palavra "mídias" pode estar relacionada à não diferenciação entre os termos "mídias sociais" e "redes sociais" por usuários e pesquisadores. Os resultados indicam que, no caso do Twitter, os trabalhos encontrados sempre estavam associados a mais de uma rede social, o que pode estar relacionado ao fato de o Twitter ser uma ferramenta que permite conectividade com outras redes sociais. Por sua vez, a palavra "Instagram" não apareceu em nenhum dos trabalhos pesquisados, mas acredita-se ser uma rede social em ascensão, com capacidade de atrair estudos futuros.

Ainda com relação aos artigos selecionados para análise, observou-se o surgimento das pesquisas nesta área a partir de 2007, o que pode estar associado ao fato do Orkut ter surgido no Brasil em 2004, chegando a atingir 30 milhões de usuários em 2014, ano do seu encerramento (Abril, 2014). Já o Facebook conquistou, no mesmo ano, 89 milhões de usuários no Brasil (Uol, 2014). O ano de 2014 foi o em que mais pesquisas foram publicadas sobre o tema pesquisado; acredita-se que a tendência é que aumente o número de pesquisas a este respeito nos próximos anos, devido ao aumento da curiosidade sobre o assunto e da sua presença na vida das pessoas. Inclusive, vislumbra-se a realização de estudos sobre mídias sociais mais recentes, como o Instagram, Pinterest, WhatsApp, entre outras.

Constatou-se que a maioria dos artigos analisados é de estudos exploratórios $(56,9 \%)$, resultado que sugere que ainda se busca a construção mais consistente da temática que relaciona redes sociais e comportamento do consumidor na academia brasileira. Esse fato justifica-se uma vez que as pesquisas a esse respeito são recentes, compreendendo um período de 9 anos, e o intuito é a busca de um entendimento inicial desse fenômeno no tocante ao comportamento do consumidor. Outro fato que se relaciona com a quantidade de estudos exploratórios são os estudos empíricos, que são maioria $(94,5 \%)$, e que representaram pesquisas baseadas em experimentação, experiência vividas e observações. Também relacionado a isso está o fato dos estudos de casos $(44,4 \%)$ e survey $(24,1 \%)$ terem sido maioria nos métodos de coleta de dados, priorizando dados primários $(70,4 \%)$.

Diante das exigências por parte das publicações relevantes que os artigos apresentem contribuições para o público leitor e para as organizações, analisaramse os trabalhos individualmente, notando-se que um grande número não apresenta recomendações para pesquisas futuras $(46,3 \%)$ e recomendações para aplicação 
prática $(63,0 \%)$. Esse fato sugere pontos de melhoria para novos trabalhos sobre 0 tema.

Também se verificou uma variedade dos segmentos de consumidor pesquisados, sem uma concentração clara em algum desses grupos. O que mais foi discutido nos artigos são os usuários, de forma geral, de redes sociais. No entanto, outros segmentos foram percebidos, o que denota que o uso de redes sociais e o comportamento das pessoas que usam essas redes é bastante amplo, ou seja, as pessoas divulgam suas dúvidas, reclamam de determinados serviços, produtos, empresas, pedem indicações, entre outras possibilidades.

A pesquisa possibilitou a observação das relações entre organização e indivíduo nas redes sociais, o que trouxe como descoberta que as redes sociais estão sendo utilizadas por consumidores para mostrar seus pensamentos e atitudes em relação às organizações, bem como o que as empresas fazem com relação aos consumidores. Nesse sentido, pode-se observar que a maioria dos trabalhos trata de pesquisas que consideram a relação indivíduo-organização, ou seja, como os indivíduos manifestam suas opiniões a respeito das organizações. Outras formas de relacionamento também foram encontradas, como organização-indivíduo e análises somente dos indivíduos ou organizações, porém em menor número, evidenciando uma lacuna a ser suprida, gerando a possibilidade de pesquisas que tratem dessas formas de relacionamento.

Contudo, um importante tipo de triangulação não foi encontrado em nenhum trabalho: organização-indivíduo-organização. Acredita-se que esse tipo de relação é de grande importância para os gestores, pois permite que as organizações e os consumidores estabeleçam um canal de comunicação direto e com feedback, o que já é relativamente comum de se constatar atualmente. Portanto, acredita-se que essa lacuna pode ser aproveitada pelos pesquisadores da área.

Este trabalho apresenta algumas limitações. Uma delas refere-se à escolha dos artigos analisados, visto que outros periódicos com qualificações diferentes no sistema WebQualis das analisadas neste estudo podem ter publicações a respeito do tema; ademais, também se optou por não analisar os artigos publicados em eventos nacionais.

Acredita-se que este trabalho apresenta como contribuição à academia a possibilidade de se aperfeiçoarem as publicações sobre o tema pesquisado, com estudos mais completos, que abordem suas contribuições efetivas para a prática e a 
teoria, bem como apresentando avanços em relação aos demais já publicados sobre o assunto. Pode-se, também, buscar compreender melhor a realidade das pesquisas publicadas sobre os dois temas pesquisados - redes sociais e comportamento do consumidor.

Como indicação para estudos futuros, propõem-se estudos bibliométricos incluindo periódicos internacionais, podendo-se estender a pesquisa para periódicos não analisados neste estudo (como os estratos B3, B4, B5 do sistema WebQualis) e congressos da área, como o EnANPAD e EMA.

Também para pesquisas futuras sugerem-se temas relacionados à triangulação da relação organização-indivíduo-organização e indivíduo-organizaçãoindivíduo, pois acredita-se que esse é um importante campo dentro da temática do comportamento do consumidor nas redes sociais, e que pode ajudar as organizações em melhorias no relacionamento com seus clientes e a estabelecerem ações de marketing mais assertivas, bem como auxiliar as pessoas a terem respostas mais efetivas por parte das organizações às suas solicitações e sugestões.

\section{Referências}

Abril. Orkut completa 10 anos com 6 milhões de brasileiros ainda ativos. Recuperado em 25 de janeiro, 2015, de http://info.abril.com.br/noticias/tecnologiapessoal/2014/01/orkut-completa-10-anos-com-6-milhoes-de-brasileiros-aindaativos.shtml

Amaral, B. do (2014). Mundo terá 3 bilhões de usuários de Internet até o final de 2014, diz UIT. Recuperado em 01 de setembro, 2014, de http://www.teletime.com.br/05/05/2014/mundo-tera-3-bilhoes-de-usuarios-deinternet-ate-o-final-de-2014-diz-uit/tt/376521/news.aspx

Bertoncello, s. T., \& Crescitelli, E. (2009). Comportamento do jovem consumidor mediante as diversas alternativas de mídia. Revista Brasileira de Pesquisas de Marketing, Opinião e Mídia, 3.

Blessa, R. (2006). Merchandising no ponto-de-venda. São Paulo: Atlas.

Capes, (2012). Web Qualis. Recuperado em 14 de fevereiro, 2014, de http://qualis.capes.gov.br/webqualis/ConsultaListaCompletaPeriodicos.faces

Caputo, V. (2014). Mais da metade dos brasileiros são usuários da internet. Recuperado em 01 de setembro, 2014, de 
http://exame.abril.com.br/tecnologia/noticias/mais-da-metade-dos-brasileiros-saousuarios-da-internet

Chauvel, M. A. (1999). A satisfação do consumidor no pensamento de marketing: revisão de literatura. Anais do 23ํㅡㄹ Encontro Nacional da Associação Nacional de Pós Graduação em Administração. Foz do Iguaçu, PR, Brasil.

Cobra, M. (1993). Marketing competitivo: uma abordagem estratégica. São Paulo: Atlas.

Fagundes, A. F. A., Veiga, R. T., Sampaio, D. O., \& Sousa, C. V. (2012). A publicação acadêmica de marketing esportivo no Brasil. Revista Brasileira de Marketing, 11(2), 96-123.

Flick, U. (2004). Uma introdução à pesquisa qualitativa (Vol. 2). Porto Alegre: Bookman.

Frio, R. S. (2014). A pesquisa sobre comportamento do consumidor no Brasil: uma análise bibliométrica acerca de artigos publicados em periódicos nacionais no período de 2000 a 2011. Revista de Administração e Negócios da Amazônia, 5(3), 1-14.

Gomes, H. S. (2013). Brasil é o 2o país com mais usuários que entram diariamente no Facebook. Recuperado em 18 de outubro, 2014, de http://g1.globo.com/tecnologia/noticia/2013/09/brasil-e-o-2-pais-com-mais-usuarios-queentram-diariamente-no-facebook.html

Guedes, V. L., \& Borschiver, S. (2005). Bibliometria: uma ferramenta estatística para a gestão da informação e do conhecimento, em sistemas de informação, de comunicação e de avaliação científica e tecnológica. Anais do 6o Encontro Nacional de Ciência da Informação, p. 1-18.

Guimarães, M. D. S. (2011). Relações Públicas e Mídias Sociais: uma análise de suas aplicações no relacionamento organizacional. (Dissertação Mestrado em Ciências da Comunicação. Universidade Federal do Amazonas - UFAM - AM, Brasil).

Hercheui, M. D. (2011). Redes sociais: uma nova perspectiva do espaço. GVexecutivo, 10(2), 67.

Jansen, T. (2014). Número de internautas no Brasil alcança percentual inédito, mas acesso ainda é concentrado. Recuperado em 01 de setembro, 2014, de http://oglobo.globo.com/sociedade/tecnologia/numero-de-internautas-no-brasilalcanca-percentual-inedito-mas-acesso-ainda-concentrado-13027120\#ixzz3C4JzJvEE

Kimura, H., Basso, L. F. C., \& Martin, D. M. L. (2008). Redes sociais e o marketing de inovações. Revista de Administração Mackenzie, 9(1).

Kleinubing, L. S. (2010). Análise bibliométria da produção científica em gestão da informação na base de dados Lisa. Revista Digital de Biblioteconomia e Ciência da Informação, 8(1), 01-11. 
Kotler, P., \& Armstrong, G. (2002). Administração de Marketing: a edição do novo milênio. São Paulo: Prentice Hall.

Kotler, P., \& Armstrong, G. (1995). Princípios de marketing (5a ed.). Rio de Janeiro: Prentice-Hall do Brasil.

Laudon, K. C., \& Laudon, J. P. (2004). Sistemas de informação gerenciais. São Paulo: Prentice Hall.

Lemes, L. B., \& Ghisleni, T. S. (2013). Marketing Digital: uma estratégia de relacionamento de marca. Anais do 14ํㅡㄹ Congresso de Ciências da Comunicação na Região Sul, Santa Cruz do Sul, RS, Brasil.

Lima, M. P., Kraemer, F., \& Rossi, C. A. V. (2014). A discussão epistemológica em marketing 1990-2010. Revista Brasileira de Marketing, 13(1), 133-143.

Limeira, T. M. V. (2000). E-marketing: O Marketing na Internet com Casos Brasileiros. São Paulo: Editora Saraiva.

Machado-Da-Silva, C. L., Cunha, V. C., \& Amboni, N. (1990). Organizações: o estado da arte da produção acadêmica no Brasil. Anais 14ํㅡㄹ Encontro Nacional da Associação Nacional de Pós-Graduação e Pesquisa em Administração, Florianópolis, SC, Brasil.

Marconi, M. de A., \& Lakatos, E. M. (2007). Fundamentos de Metodologia Científica (6a ed.). São Paulo: Atlas.

Maya, P. C., \& Otero, W. I. (2002). A Influência do Consumidor na era da Internet. Revista da FAE, 5(1), 71-81.

Mazzon, J. A., \& Hernandez, J. M. (2013). Produção científica brasileira em Marketing no período 2000-2009. Revista de Administração de Empresas, 53(1), 67-80.

Muylder, C. F. De, Santos, M. F., La Falce, J. L., de Britto, E., \& Cançado, V. L. (2012). Principais Aplicações de Análise Discriminante na Área de Marketing: uma pesquisa bibliométrica. Revista Gestão \& Tecnologia, 12(2), 217-242.

Perin, M., Sampaio, C., Froemming, L., \& Luce, F. (2000). A perspectiva survey em artigos de marketing nos ENANPADs da década de 90. Anais do 24 Encontro Nacional de Programas de Pós Graduação em Administração, Florianópolis, SC, Brasil.

Pinto, M. R., \& Lara, J. E. (2009). O que se publica sobre comportamento do consumidor no Brasil, afinal?. Revista de Administração da UFSM, 1(3).

Recuero, R. (2011). Redes sociais na internet. Porto Alegre:Sulina.

Rossi, C. A. V., Bortoli, L. V., \& Castilhos, R. B. (2014). Análise bibliométrica da contribuição de marketing para outras ciências. Revista de Ciências da Administração, 29-44. 
Samara, B. S., \& Morsch, M. A. (2005). Comportamento do consumidor: conceitos e casos. São Paulo: Prentince Hall.

Sampaio, D. de, Gosling, M., Fagundes, A. F. A., \& Veloso, C. (2013). Uma Análise da Produção Acadêmica Brasileira sobre o Comportamento do Consumidor de Alimento Orgânico entre 1997 a 2011, ReAd. 76(3), 620-645.

Scharf, E. R., Schwingel, A. T., \& Franzon, F. (2013). Um Estudo Bibliométrico Sobre a Produção Científica de Marketing: O Final da Primeira Década dos Anos 2000. Visão, 2(1), 5-26.

Souza, M. D. (2010). O neoconsumidor e os canais de venda. Revista HSM Management, (78).

Stanley, C. (2009). A web 2.0 e o marketing das redes sociais. Revista HSM Management, 758.

Telles, A. (2011). A revolução das mídias sociais: estratégias de marketing digital para você e sua empresa terem sucesso nas mídias sociais: cases, conceitos, dicas e ferramentas. São Paulo: M. Books.

Torres, C. (2009). A bíblia do marketing digital. São Paulo: Novatec.

Troccoli, I. R., Gimenez, K., Reis, P. N. C., \& Faria, P. P. (2011). Comportamento do consumidor: Um estudo bibliométrico nos Enanpads 2007-09. Revista Organizações em Contexto-Online, 7(14), 165-189.

Turchi, S. (2013). Os novos comportamentos de consumidores e marcas com o advento das redes sociais. Recuperado em 26 e outubro, 2014, de http://www.sandraturchi.com.br/artigos/08/os-novos-comportamentos-dconsumidores-e-marcas-com-o-advento-das-redes-sociais/

UOL. 89 milhões de brasileiros acessam o Facebook. Recuperado em 25 de janeiro, 2015, de http://m.olhardigital.uol.com.br/noticia/89-milhoes-de-brasileiros-acessam-ofacebook/43687

Vaz, C. A. (2008). Google Marketing. São Paulo: Novatec.

Vaz, C. A. (2012). Os 8ps do Marketing digital: o guia estratégico de Marketing digital. Alfragide: Texto editores.

Vergara, S. C. (2009). Projetos e relatórios de pesquisa em administração. São Paulo: Atlas.

Vieira, F. G. (1998). Por quem os sinos dobram? Uma análise da publicação científica na área de marketing do Enanpad. Anais do $22^{\circ}$ Encontro Nacional da Associação Nacional de Pós-Graduação e Pesquisa em Administração, Foz do Iguaçu, PR, Brasil .

Vieira, F. G. (1999). Ações empresariais e prioridades de pesquisa em marketing: tendências no Brasil e no mundo segundo a percepção dos acadêmicos 
brasileiros. Anais do $22^{\circ}$ Encontro Nacional de Programas de Pós-graduação em Administração, Foz do Iguaçu, PR, Brasil.

Vieira, F. (2003). Narciso sem espelho: a publicação brasileira de Marketing. Revista de Administração de Empresas, 43(1), 81-90.

Vieira, F. (2000). Panorama acadêmico-científico e temáticas de estudos de Marketing no Brasil. Anais do $24^{\circ}$ Encontro Nacional da Associação Nacional de Pós-Graduação e Pesquisa em Administração, Florianópolis, SC, Brasil. 